\title{
THE DOUBLE POINT FORMULA WITH ISOLATED SINGULARITIES AND CANONICAL EMBEDDINGS
}

\author{
FABRIZIO CATANESE, KEIJI OGUISO
}

\begin{abstract}
Motivated by the embedding problem of canonical models in small codimension, we extend Severi's double point formula to the case of surfaces with rational double points, and we give more general double point formulae for varieties with isolated singularities.

A concrete application is for surfaces with geometric genus $p_{g}=5$ : the canonical model is embedded in $\mathbb{P}^{4}$ if and only if we have a complete intersection of type $(2,4)$ or $(3,3)$.
\end{abstract}

\section{Contents}

1. Introduction

2. The classical double point formula

3. Extension of Severi's Statement

4. Comparing normal sheaf and quotient bundle

5. Surfaces with rational double points

6. Double point formulae via symplectic approximations.

References

2010 Mathematics Subject Classification. 14J29, 14J17, 14N15, 14E 99, 14B05, 32C22, 32Q40, 57R12, 57R42.

Key words and phrases. Improper double points, double point formula, canonical models of surfaces, rational double points, isolated singularities, Chern classes, Euler number, symplectic smoothing, tangent star, Gauss map.

The first author acknowledges support of the ERC 2013 Advanced Research Grant 340258 - TADMICAMT. The second author acknowledges support of JSPS Grant-in-Aid (S) 15H05738, JSPS Grant-in-Aid (B) No 15H03611. 


\section{INTRODUCTION}

Already for irreducible plane curves $C \subset \mathbb{P}_{\mathbb{C}}^{2}$ of degree $d$ with $\delta$ ordinary double points (i.e., with local analytical equation $x y=0$ ) we have two types of double point formulae.

First there is the Plücker formula for the class $\omega_{1}(C)$, which is a projective invariant, the number of lines $L$ passing through a general point $O \in \mathbb{P}^{2}$ and tangent to the curve $C$ at some smooth point:

$$
\text { (I) } \omega_{1}(C)=d(d-1)-2 \delta,
$$

second, there is the genus formula:

$$
\text { (II) } 2 g(C)-2=d(d-3)-2 \delta,
$$

where $2 \mathrm{~g}(\mathrm{C})$ is the first Betti number of the normalization $\tilde{C}$ of $C$, which is a topological invariant.

The two formulae can be rewritten as expressing the number of double points in terms of the degree and the class, respectively in terms of the degree and the genus:

$$
2 \delta=d(d-1)-\omega_{1}(C)=d(d-3)+2-2 g(C) .
$$

Both formulae can be generalized also for plane curves with more general singularities, but the number $2 \delta$ is replaced differently in each of the two formulae.

In the genus formula, the number $2 \delta$ is replaced by the sum

$$
\sum_{P} m_{P}\left(m_{P}-1\right)
$$

where the sum runs over all points $P$, also the infinitely near ones, and $m_{P}$ is the multiplicity of (the strict transform of) the curve $C$ in $P$.

In the Plücker formula appear not only the multiplicity of a singular point, but also the local classes of the branches of $C$ at the singular points (cf. GH78, Pages 277-282]), and, for instance, in the case of an ordinary cusp singularity, i.e., when the local analytic equation of $C$ is

$$
y^{2}-x^{3}=0,
$$

the contribution for the Plücker formula is 3 , the sum of the multiplicity (= 2 ) and the local class $(=1)$.

Another 'philosophical' aspect of the genus formula is that it explains that a smooth projective curve of genus $g$ and degree $d$ cannot be projected isomorphically to the plane $\mathbb{P}^{2}$ if

$$
2 g-2-d(d-3) \neq 0 .
$$

It was classically known that every smooth subvariety $X^{\prime} \subset \mathbb{P}^{N}$ of dimension $n$ can be projected isomorphically to $X \subset \mathbb{P}^{2 n+1}$, since the secant variety of $X^{\prime}$ has dimension $2 n+1$ and does not fill up the whole space if $N \geq 2 n+2$, so that we can project $X$ from a general point $O$ isomorphically in $\mathbb{P}^{N-1}$. But when you want to project to $\mathbb{P}^{2 n}$ there appear, for a general projection, $\delta$ Improper Double Points $=$ IDP 's , that is isolated singularities consisting of two smooth branches intersecting transversally. 
If we put ourselves in a situation similar to the one of plane curves with only IDP's as singularities (in this case IDP's are the same as before, ordinary double points), again we have two double point formulae.

The following Severi's double point formula, established by Severi in 1902, is a generalization of the Plücker formula:

$$
\text { (I) } 2 \delta=d(d-1)-\sum_{i=1}^{n} \omega_{i}(X),
$$

and is given in terms of projective invariants $\omega_{i}(X)$, called the ceti of $X$ (see section 2).

Later on, it was realized that these projective invariants are related to absolute invariants (similar to the genus $g$ of a curve), and this led to the theory of characteristic classes, notably Chern classes. In terms of these, we have the Todd-Fulton-Laksov formula, which is the modern double point formula:

$$
\text { (II) } 2 \delta=d^{2}-c_{n}\left(N_{f}\right)=d^{2}-\left[c\left(T_{X^{\prime}}\right)^{-1}(1+H)^{2 n+1}\right]_{n},
$$

where $f: X^{\prime} \rightarrow X$ is the normalization map, $N_{f}$ is the normal bundle to the immersion $f$, defined through the exact sequence

$$
0 \rightarrow T_{X^{\prime}} \rightarrow f^{*}\left(T_{\mathbb{P}^{2 n}}\right) \rightarrow N_{f} \rightarrow 0,
$$

where we denote by $H$ the pull back of the hyperplane class to $X^{\prime}$, and where $\left[c_{0}+c_{1}+\cdots+c_{n}\right]_{n}$ denotes the part $c_{n}$ of dimension $2 n$ of the sum in the cohomology ring (or in the Chow ring) of $X^{\prime}$.

This double point formula has, as we saw, two formulations (the second follows from multiplicativity of the Chern classes for exact sequences); the first has a topological flavour, and allows for generalizations: it says that $d^{2}$, the self intersection number of $X$ in $\mathbb{P}^{2 n}$, equals to $2 \delta$ plus the self intersection number of the zero section in the normal bundle $N_{f}$ (which maps via the exponential map onto a neighbourhood of $X$ ).

The second instead lends itself to concrete calculations, by which it is possible to exclude that a smooth variety $X^{\prime}$ of dimension $n$ can be embedded in a projective space of dimension $\leq 2 n$ by certain linear systems.

For instance, the first author has proven elsewhere [Ca19] the following result:

Theorem 1.1. Let $D$ be an ample smooth divisor in an Abelian variety $A$ of dimension $n+1$. If $D$ yields a polarization of type $\left(m_{1}, \ldots, m_{n+1}\right)$, then the canonical map $\Phi_{D}$ of $D$ is a morphism, and it can be an embedding only if $p_{g}(D):=h^{0}\left(K_{D}\right) \geq 2 n+2$, which means that the Pfaffian $m:=$ $m_{1} \cdot m_{2} \cdots m_{n+1}$ satisfies the inequality

$$
m \geq n+2 .
$$

The main idea for proving the above theorem is, assuming that $D$ is embedded by $H^{0}\left(K_{D}\right)=H^{0}\left(\mathcal{O}_{D}(D)\right)$ and that $N:=p_{g}(D)-1 \leq 2 n$, to apply the double point formula (in the special case $\delta=0$ ) to $D$ and to its iterated hyperplane sections. See also deV75, Ka19. for other applications.

The main motivation for our work stems from surface theory, namely the desire to extend the following theorem of the first author [Ca97]: 
Theorem 1.2. Let $S$ be a complex projective surface with ample canonical divisor and with $p_{g}(S)=5$. Then the canonical map is an embedding if and only $S$ is a smooth complete intersection in $\mathbb{P}^{4}$ of type $(2,4)$ or of type $(3,3)$.

If we take instead a minimal surface $S$ of general type with $p_{g}(S)=5$, the canonical map factors through the canonical model $S^{\prime}$ of $S$, a normal surface with canonical singularities (rational double points), and we may ask the similar question: when do we get an embedding of the canonical model? The answer is analogous:

Corollary 1.1. Let $S$ be a minimal smooth surface of general type with $p_{g}(S)=5$ such that the canonical map

$$
\Phi_{\left|K_{S}\right|}: S \rightarrow S^{\prime} \subset \mathbb{P}^{4}
$$

is a morphism with image isomorphic to the canonical model $S^{\prime}$ of $S$. Then, setting $d:=\left(K_{S}^{2}\right)$,

$$
12 \chi\left(\mathcal{O}_{S}\right)=(17-d) d .
$$

In particular, $S^{\prime}$ is then a complete interesection of type $(2,4)$ or $(3,3)$, if either the base field is of characteristic 0 or the characteristic of the field $k$ is $\neq 2$ and $h^{1}\left(\mathcal{O}_{S}\right)=0$.

The idea is to extend the double point formula to the case of surfaces with singularities either IDP's or canonical singularities (and then setting $\delta=0$ for the application): this is done in an elementary way in Theorem 5.1. Section 5 over any algebraically closed field of any characteristic. Then one establishes the above numerical formula, by which follows that $d=8,9$; then the proof proceeds exactly as in Theorem 1.2.

Since it would be interesting to extend this type of result also in higher dimension, it is desirable to extend the double point formula to varieties $X$ with singularities. In this paper we restrict to varieties with isolated singularities.

Fulton and Laksov's generalization [FL77] allows in particular the variety $X$, outside the IDP' $\mathrm{s}$, to have local complete intersection singularities, a property which implies that $X$ is locally smoothable at these points.

In this flavour we prove in the last section another generalization, of which the following theorem is a special case:

Theorem 1.3. Let $X \subset \mathbb{P}^{2 n}$ be a variety with isolated singularities, of which $\delta$ are Improper Double Points, and the other are normal singularities admitting a smoothing. Then $X$ admits a global smoothing to a symplectic immersed manifold $M \subset \mathbb{P}^{2 n}$, with exactly $\delta$ Improper Double Points, and we have, if $f: M^{\prime} \rightarrow M$ is the immersion,

$$
d^{2}=2 \delta+e\left(N_{f}\right),
$$

where $e$ is the Euler class of the oriented normal bundle to the map.

In spite of their elegance, the two above generalizations have the drawback that the top Chern class of the normal bundle of the partial resolution of $X$ at the IDP's (respectively the Euler class in Theorem 1.3) is not directly computable (at least this is our impression). This is due to the fact that Chern classes are multiplicative only for sheaves on smooth varieties, or, 
for general varieties, only for exact sequences of vector bundles, while Euler classes are not multiplicative.

It seems therefore worthwhile to go back to Severi's approach.

Severi established an inductive formula,

$$
2 \hat{\delta}=2 \delta+\omega_{n}(X),
$$

where $\hat{X}$ is obtained from $X$ intersecting with a general hyperplane $H$, and then projecting $X \cap H$ from a general point $O \in H$ to $\mathbb{P}^{2 n-2}$, to obtain $\hat{X}$ with $\hat{\delta}$ IDP's.

First of all we explain in Section 2 how the two different double point formulae are related to each other, showing how to formulate Severi's inductive formula in terms of the Gauss map of the immersion $f: X^{\prime} \rightarrow \mathbb{P}^{2 n}$.

Indeed, if $Q_{X^{\prime}}$ is the pull back of the universal quotient bundle of the Grassmannian $\operatorname{Gr}(n, 2 n)$ (of projective subspaces of dimension $n$ in $\mathbb{P}^{2 n}$ ), then we have:

$$
\omega_{n}(X)=c_{n}\left(Q_{X^{\prime}}\right),
$$

and the relation between the two approaches is given by the simple formula

$$
Q_{X^{\prime}}=N_{f}(-1) \text {. }
$$

For worse singularities, we take a resolution $Y$ of $X$ on which the Gauss map becomes a morphism. Then $f: Y \rightarrow \mathbb{P}^{2 n}$ is no longer an immersion and the two sheaves are different, yet there is a surjection $N_{f}(-1) \rightarrow Q_{Y}$, and the double point formula can be expressed in terms of the kernel sheaf $\mathcal{F}$, called discrepancy sheaf, see Theorem 4.1.

This is done in Section 4, using the results of Section 3 where we generalize Severi's double point formula by extending Severi's inductive formula almost verbatim, but using the notion of the tangent star of a singular point $x$ : this is the closure of the union of the lines which are limits of secant lines joining a pair of points $x_{1}, x_{2}$ tending to $x$.

Theorem 1.4. Let $X \subset \mathbb{P}:=\mathbb{P}^{2 n}$ be an irreducible nondegenerate subvariety of dimension $n$ having isolated singularities $x_{1}, \ldots, x_{h}$.

Assume that these singularities are either Improper Double Points or have tangent star of dimension $\leq 2 n-1$.

Let $\delta$ be the number of the IDP' $\mathrm{s}$ : then, in the notation of Theorem 2.1. we have

$$
2 \hat{\delta}=2 \delta+\omega_{n}(X) .
$$

Of course our above partial results raise several questions.

First of all, in Theorem 3.3 we observe that IDP's have full tangent star (the tangent star is the whole space).

There is an obvious generalization of Theorem 3.3. for varieties with isolated singularities: but the main problem here is to calculate exactly the contribution of the points with full tangent star to the double point formula. This problem is related to work of Flenner, O' Carroll and Vogel and others, who defined a cycle which gives (as far as we understand) the contribution to this multiplicity see [FOCV99]. 
This said, the topic of double point formulae can been treated in so many different ways, that it is a very challenging and currently active area of research to investigate the relations among the several approaches: our approach here was led by the problem we wanted to solve, and by the search of effectively computable formulae.

The expert reader may notice that the resolution of indeterminacy of the Gauss map was later called Nash blowing up, and used by Mather to define Chern classes of singular varieties Na95, Ma74. However, Mather-Chern classes are defined by taking the push forward of the Chern classes of the pull back (under the Gauss map) of the universal subbundle, whereas we need those of the quotient bundle to be pushed forward, and again we are missing multiplicativity. MacPherson Ma74 introduced functorial Chern classes of singular varieties; Piene Pie88 obtained expressions relating the polar classes to the Chern-Mather classes, and used these to calculate local Euler obstructions of hypersurfaces in terms of Milnor numbers. Recently Aluffi related the MacPherson Chern classes to the Fulton Chern classes first [A194 in the case of hypersurfaces, and later, Al18], in greater generality. There has been other related recent work, such as [Al16], BSS09].

It would be interesting to see whether some of these ideas shed some light on the problem of embeddings of canonical models in higher dimension, but we postpone this investigation to the future.

\section{The Classical Double Point Formula}

The classical double point formula established by Severi in 1902 concerns the following situation:

$X$ is an irreducible subvariety of dimension $n$ in $\mathbb{P}^{2 n}$, whose singularities are only Improper Double Points = IDP 's , that is isolated singularities consisting of two smooth branches intersecting transversally.

Letting $X^{\prime}$ be the normalization of $X, X^{\prime}$ is smooth projective, and we have a normalization map $\nu: X^{\prime} \rightarrow \mathbb{P}^{2 n}$ which is an immersion, hence we have the Gauss map

$$
\gamma: X^{\prime} \rightarrow G r(n, 2 n)
$$

associating to $x \in X^{\prime}$ the projective linear subspace image of the tangent space $T X_{x}^{\prime}$ under the derivative $D \nu_{x}$ of $\nu$ at $x$.

Definition 2.1. The n-th ceto $\omega_{n}(X)$ of $X \subset \mathbb{P}^{2 n}$ is the number of subspaces $\gamma(x)$ passing through a general point $O \in \mathbb{P}^{2 n}$.

More generally, for any projective variety $X$ of dimension $n$ in $\mathbb{P}^{N}, X \subset$ $\mathbb{P}^{N}$, the $\mathrm{n}$-th ceto of $X, \omega_{n}(X)$, is the number of linear subspaces of dimension $n$ tangent to $X$ at smooth points, and intersecting a general linear subspace of codimension $2 n$ in $\mathbb{P}^{N}$.

The i-th ceto of $X, \omega_{i}(X)$, is the i-th ceto of the intersection $X \cap L^{\prime}$ of $X$ with a general subspace $L^{\prime}$ of codimension $n-i$.

The following is Severi's main assertion ([Se02], see also [Ca79] for a more detailed proof)

Theorem 2.1. (Severi' $\mathbf{s}$ Inductive Statement) Let $X \subset \mathbb{P}^{2 n}$ be a subvariety of dimension $n$ having only $\delta$ IDP' s as singularities, let $O \in \mathbb{P}^{2 n}$ 
be a general point, let $L$ be a general linear subspace of $\mathbb{P}^{2 n}$ of codimension 2 , let $H$ be the hyperplane spanned by $O$ and $L$.

Then, denoting by $\hat{X}^{\prime}$ the (smooth) intersection $X \cap H$, the projection with centre $O$ to $L$ of $\hat{X}^{\prime}$ is a variety $\hat{X}$ with only $\hat{\delta}$ IDP' s as singularities, and we have

$$
2 \hat{\delta}=2 \delta+\omega_{n}(X)
$$

Using the inductive statement, and observing that, for a plane curve of degree $d$ and with $\delta^{\prime}$ double points, the 1-ceto is just its class, the number of tangents through a general point, hence equal to $d(d-1)-2 \delta^{\prime}$, Severi obtains the following result:

Theorem 2.2. (Severi' s Double Point Formula) Let $X \subset \mathbb{P}^{2 n}$ be a subvariety of dimension $n$ and degree $d$ having only $\delta$ IDP's as singularities:

then we have

$$
2 \delta=d(d-1)-\sum_{i=1}^{n} \omega_{i}(X) .
$$

The modern version of the double point formula uses holomorphic vector bundles and Chern classes, and can be geometrically explained as follows, over the complex numbers.

Define the normal bundle $N_{\nu}$ to the map $\nu$ through the exact sequence associated to the derivative of $\nu$

$$
0 \rightarrow T_{X^{\prime}} \rightarrow \nu^{*}\left(T_{\mathbb{P}^{2 n}}\right) \rightarrow N_{\nu} \rightarrow 0 .
$$

The exponential map yields an oriented submersion of a neighbourhood of the 0-section of $N_{\nu}$ (which we identify to $X^{\prime}$ ) onto a neighbourhood of $X$, and one can find a small perturbation of the 0 -section yielding an oriented submanifold $X_{t}^{\prime}$ intersecting $X^{\prime}$ transversally in a finite number of points, so that the self intersection $\left(X^{\prime}\right)^{2}$ of $X^{\prime}$ in $N_{\nu}$ equals the Euler class of the bundle $N_{\nu}$, in turn equal to the top Chern class $c_{n}\left(N_{\nu}\right)$.

It is easy to see that the self intersection of $X$ equals $X^{2}=2 \delta+\left(X^{\prime}\right)^{2}$ : since $X$ has degree $d$, we get (see [LMS75] for an algebraic proof)

Theorem 2.3. (Modern Double Point Formula) Let $X \subset \mathbb{P}^{2 n}$ be a subvariety of dimension $n$ and degree $d$ having only $\delta$ IDP' $\mathrm{s}$ as singularities:

then we have

$$
(D P) d^{2}=2 \delta+c_{n}\left(N_{\nu}\right)=2 \delta+c_{n}\left(\nu^{*}\left(T_{\mathbb{P}^{2 n}}\right)-T_{X^{\prime}}\right) .
$$

Using then the multiplicativity of Chern classes for exact sequences, and that the total Chern class $c\left(T_{\mathbb{P}^{2 n}}\right)=c(\mathcal{O}(1))^{2 n+1}$, if we denote by $H$ the pull back of the hyperplane class to $X^{\prime}$, we get

$$
2 \delta=d^{2}-\left[c\left(T_{X^{\prime}}\right)^{-1}(1+H)^{2 n+1}\right]_{n},
$$

where $\left[c_{0}+c_{1}+\cdots+c_{n}\right]_{n}$ denotes the part $c_{n}$ of dimension $2 n$ of the sum in the cohomology ring (or in the Chow ring).

The most interesting (not only historical) question is then: how are the two formulae related? 
One deals with projective invariants of $X$, the other with invariants of the abstract variety $X^{\prime}$, but indeed the Gauss map $\gamma$ produces some vector bundles on $X^{\prime}$.

Denote as usual by $V$ the complex vector space such that $\mathbb{P}(V)=\mathbb{P}^{2 n}$ (here $\mathbb{P}(V)$ is the variety of 1-dimensional subspaces of $V$ ): then on the Grassmann variety $G:=\operatorname{Gr}(n, 2 n)$ we have an exact sequence of vector bundles

$$
0 \rightarrow U \rightarrow V \otimes \mathcal{O}_{G} \rightarrow Q \rightarrow 0,
$$

where $U$ is the universal subbundle, whose fibre at a subspace $W \subset V$ is tautologically equal to $W$, while $Q$ is the quotient bundle.

In our situation, a point $O \in \mathbb{P}:=\mathbb{P}(V)$ is the class $[v]$ of a vector $v \in V \backslash\{0\}$, and $v$ defines a regular section of $V \otimes \mathcal{O}_{G}$, and, by composition, $s_{v}: \mathcal{O}_{G} \rightarrow Q$. The section $s_{v}$ vanishes exactly at the subspaces $W$ which contain $v$.

Taking the pull-back under the Gauss map, we get

$$
0 \rightarrow \gamma^{*} U \rightarrow V \otimes \mathcal{O}_{X^{\prime}} \rightarrow \gamma^{*} Q \rightarrow 0,
$$

and we see then that $\omega_{n}(X)$ equals to the numbers of zeroes of $\gamma^{*}\left(s_{v}\right)$, hence

$$
\omega_{n}(X)=c_{n}\left(\gamma^{*} Q\right) .
$$

The Euler sequence in $\mathbb{P}(V)=: \mathbb{P}$ pulls back to $X^{\prime}$ yielding

$$
0 \rightarrow \nu^{*} \mathcal{U} \rightarrow V \otimes \mathcal{O}_{X^{\prime}} \rightarrow \nu^{*} T_{\mathbb{P}}(-1) \rightarrow 0 .
$$

Here $\mathcal{U} \cong \mathcal{O}_{\mathbb{P}}(-1)$ is the tautological subbundle on $\mathbb{P}$, and an easy but important observation is the inclusion

$$
\nu^{*} \mathcal{U} \subset \gamma^{*} U .
$$

Denoting by $\mathcal{O}_{X^{\prime}}(H):=\nu^{*} \mathcal{O}_{\mathbb{P}}(1)$, the derivative $D \nu$ yields, after twisting, the exact sequence

$$
(*) 0 \rightarrow T_{X^{\prime}}(-H) \rightarrow \nu^{*}\left(T_{\mathbb{P}}(-1)\right) \rightarrow N_{\nu}(-H) \rightarrow 0 .
$$

Moding out the other two previous exact sequences by $\nu^{*} \mathcal{U}$, we get

$$
(* *) 0 \rightarrow\left(\gamma^{*} U\right) /\left(\nu^{*} \mathcal{U}\right) \rightarrow \nu^{*}\left(T_{\mathbb{P}}(-1)\right) \rightarrow \gamma^{*} Q \rightarrow 0 .
$$

Since $\nu$ is an immersion, $D \nu_{x}: T X_{x}^{\prime} \rightarrow\left(\gamma^{*} U / \nu^{*} \mathcal{U}\right)_{x}$ is an isomorphism, hence $(*)$ and $(* *)$ are the same exact sequences. In particular, we have an isomorphism $\gamma^{*} Q \simeq N_{\nu}(-H)$.

Restricting $N_{\nu}$ to a hyperplane section $H=\hat{X}^{\prime}$, we obtain:

$$
0 \rightarrow N_{\nu}(-H) \rightarrow N_{\nu} \rightarrow N_{\nu} \mid \hat{X}^{\prime}:=N_{\nu} \otimes \mathcal{O}_{\hat{X}^{\prime}} \rightarrow 0,
$$

yielding $c\left(N_{\nu}\right)=c\left(\gamma^{*} Q\right) \cdot c\left(N_{\nu} \mid \hat{X}^{\prime}\right)$ for the total Chern classes.

The upshot here is: the restriction $N_{\nu} \mid \hat{X}^{\prime}$ is the normal bundle $N_{\nu^{\prime}}$ of the $\operatorname{map} \nu^{\prime}: \hat{X}^{\prime} \rightarrow \mathbb{P}^{2 n-1}$.

On the other hand, projection from the point $O$ yields a map

$$
\hat{\nu}: \hat{X}^{\prime} \rightarrow \mathbb{P}^{2 n-2}
$$


such that

in the K-group, hence

$$
N_{\nu^{\prime}}=N_{\hat{\nu}}+\mathcal{O}_{\hat{X}^{\prime}}(H)
$$

$$
c\left(N_{\hat{\nu}}\right)=c\left(N_{\nu^{\prime}}\right)(1+H)^{-1}
$$

in the Chow ring. We can then calculate

$$
\begin{gathered}
c_{n}\left(\gamma^{*} Q\right)=c_{n}\left(N_{\nu}(-H)\right)=c_{n}\left(N_{\nu}\right)-H c_{n-1}\left(N_{\nu}\right)+\cdots+(-1)^{n} H^{n} \\
=c_{n}\left(N_{\nu}\right)-H\left(c_{n-1}\left(N_{\nu}-\mathcal{O}_{X^{\prime}}(H)\right)\right)=c_{n}\left(N_{\nu}\right)-i_{*}\left(c_{n-1}\left(\left(N_{\nu^{\prime}}-\mathcal{O}_{\hat{X}^{\prime}}(H)\right)\right)=\right. \\
=c_{n}\left(N_{\nu}\right)-i_{*}\left(c_{n-1}\left(N_{\hat{\nu}}\right)\right)
\end{gathered}
$$

where $i: \hat{X}^{\prime} \rightarrow X^{\prime}$ is the natural inclusion.

We have thus shown

$$
\omega_{n}(X)=c_{n}\left(N_{\nu}\right)-i_{*}\left(c_{n-1}\left(N_{\hat{\nu}}\right)\right),
$$

which, using formula $(D P)$ for the double points, gives Severi's inductive formula.

\section{Extension of Severi's Statement}

In this section $X \subset \mathbb{P}:=\mathbb{P}^{2 n}$ is an irreducible nondegenerate subvariety of dimension $n$ having isolated singularities. We set $X^{*}:=X \backslash \operatorname{Sing}(X)$, and let $x_{1}, \ldots, x_{h}$ be the singular points of $X$.

Severi's basic idea starts with the consideration of the secant map

$$
\sigma:(X \times X) \backslash \Delta_{X} \rightarrow \mathcal{G}:=\operatorname{Gr}(1,2 n)
$$

associating to $x \neq x^{\prime}$ the line $\sigma\left(x, x^{\prime}\right):=x * x^{\prime}$.

Taking the closure $\Sigma$ of the graph of $\sigma$ in $(X \times X) \times \mathcal{G}$, and on it the pull-back of the projectivized universal subbundle $\mathcal{L}_{\mathcal{G}} \subset \mathcal{G} \times \mathbb{P}$ of the Grassmannian, we obtain a line incidence relation

$$
\mathcal{L}_{X} \subset \Sigma \times \mathbb{P} \subset(X \times X) \times \mathcal{G} \times \mathbb{P},
$$

which is a $\mathbb{P}^{1}$-bundle over $\Sigma$.

There is a natural surjective projection $p: \mathcal{L}_{X} \rightarrow(X \times X)$ and a natural projection $\pi: \mathcal{L}_{X} \rightarrow \mathbb{P}$.

Definition 3.1. We define as customary (cf. [Jo78]) for $x \in X$,

$$
\mathcal{L}_{x}:=p^{-1}(\{(x, x)\}),
$$

and define the tangent star of $X$ at $x$ as

$$
\operatorname{Star}_{x}(X):=\pi\left(\mathcal{L}_{x}\right)
$$

For any smooth point $x \in X$, the tangent star is the projective tangent space of $X$ at $x$, and in general one has a series of inclusions:

$$
\mathcal{T}_{x}(X) \subset \operatorname{Star}_{x}(X) \subset T^{Z a r} X_{x},
$$

in words: the tangent cone at $x$ is contained in the tangent star at $x$, which is contained in the projective closure of the Zariski tangent space.

Since $\Sigma$ has dimension $n$, the fibre $\Sigma_{x}$ over $(x, x)$ has dimension at most $2 n-1$, hence in general the tangent star at $x$ has dimension at most $2 n$. Moreover, $\Sigma_{x}$ consists of lines passing through $x$, hence 
Proposition 3.1. The tangent star is a cone with vertex $x$. If $X \subset \mathbb{P}:=\mathbb{P}^{2 n}$ has dimension $n$, then the following assertions (1), (2), (3) are equivalent:

(1) the tangent star at $x$ equals $\mathbb{P}^{2 n}$;

(2) $\Sigma_{x}$ has dimension $2 n-1$;

(3) $\Sigma_{x}$ is the Schubert cell $C_{x}$ of all lines through $x$.

In particular the degree of the map $\mathcal{L}_{x} \rightarrow \mathbb{P}^{2 n}$ is always either 0 or 1 .

Proof. Assertion (1) amounts to $\operatorname{dim}\left(\operatorname{Star}_{x}(X)\right)=2 n$, which implies $\operatorname{dim}\left(\Sigma_{x}\right) \geq 2 n-1$, which is equivalent to assertion (3) since $\Sigma_{x} \subset C_{x}=$ $\mathbb{P}^{2 n-1}$, where $C_{x}=\mathbb{P}^{2 n-1}$ is the variety (Schubert cell) of lines through $x$. Assertion (3) obviously implies (1), and also the last assertion is clear, since the degree is 0 if and only if the map is not surjective.

\section{Remark 3.2.}

(i) The IDP 's are isolated singularities with full tangent star, i.e., whose tangent star is the whole space $\mathbb{P}^{2 n}$.

(ii) Each point of embedding dimension $<2 n$ (that is, the Zariski tangent space has dimension $\leq 2 n-1$ ) has a non full tangent star.

(iii) Observe that $\mathcal{L}_{x}$ is taken here with the reduced structure: in general it is part of the ramification locus of $\pi$.

(iv) see [SUV97] for some related results concerning tangent stars of complete intersections.

We can now prove a generalization of Severi's theorem (Theorem 2.1, the inductive assertion):

Theorem 3.3. Let $X \subset \mathbb{P}:=\mathbb{P}^{2 n}$ be an irreducible nondegenerate subvariety of dimension $n$ having isolated singularities $x_{1}, \ldots, x_{h}$ only.

Assume that these singularities are either Improper Double Points or have tangent star of dimension $\leq 2 n-1$.

Let $\delta$ be the number of the IDP' $\mathrm{s}$ : then, in the notation of Theorem 2.1, we have

$$
2 \hat{\delta}=2 \delta+\omega_{n}(X) .
$$

Proof. Let $Z$ be the image of $\Sigma$ inside the Grassmannian $\mathcal{G}$. Observe that $q: \Sigma \rightarrow Z$ factors through the involution $\tau$ on $\Sigma$ birationally induced by the involution exchanging the factors of $X \times X$.

Argueing as in Lemma 1 of [Ca79], we see that $\Sigma \rightarrow Z$ is generically finite of degree 2 , hence $Z$ is irreducible of dimension $2 n$, and birational to $\Sigma / \tau$.

Given a point $O \in \mathbb{P}$, we let $C_{O}$ be the Schubert cell of the lines through $O$, and we consider the curve

$$
C:=q^{-1}\left(Z \cap C_{O}\right) \subset \Sigma \subset(X \times X) \times \mathcal{G} .
$$

Denote by $\Gamma_{2}$ the projection of $C$ inside $X \times X$, respectively by $\Gamma$ its projection to $X$ (because of $\tau$ symmetry, the first and second projection give the same result).

Observe that, by our assumption, for general choice of $O, \Gamma$ does not pass through the singular points of $X$ which are not IDP 's. 
Let $X^{\prime}$ be the normalization of $X$ precisely at the IDP 's. Then we can construct an incidence variety $\Sigma^{\prime} \subset\left(X^{\prime} \times X^{\prime}\right) \times \mathcal{G}$ which is birational to $\Sigma$. Hence we can similarly define curves $C^{\prime}, \Gamma_{2}^{\prime}, \Gamma^{\prime}$.

The proof proceeds now exactly as in Ca79, pages 766-773 (but with a slightly different notation).

Step I) Proposition 2 shows that $\Gamma_{2}^{\prime}$ is a smooth curve, and the graph of a birational involution $\tau$ on $\Gamma^{\prime}$ such that, if $x_{i}^{\prime}, x_{i}^{\prime \prime}$ are the two points lying over an IDP $x_{i}$, then $\tau\left(x_{i}^{\prime}\right)=x_{i}^{\prime \prime}$.

Step II) For general choice of $O, L$, setting $H=O * L, \hat{X}^{\prime}=X \cap H$ is smooth and $\hat{X} \subset L \cong \mathbb{P}^{2 n-2}$ has only IDP' s. Moreover $\Gamma \cap L=\emptyset, H$ intersects $\Gamma$ in $\operatorname{deg}(\Gamma)$ distinct smooth points of $\Gamma$, and different from the points $Q_{1}, \ldots, Q_{\omega_{n}(X)} 1$ where the tangent space to $X$ does not pass through $O$, so that in particular $\tau$ has no fixed points on $H \cap \Gamma$. Denote these points by $R_{1}, \ldots, R_{2 m}$. We observe that $m=\hat{\delta}$ : since the points in $\Gamma \cap H$ contribute in pairs to IDP's of $\hat{X}$.

Step III ) Let $\phi_{0}, \phi_{1}$ be linear forms such that $H=\left\{\phi_{0}=0\right\}, L=\left\{\phi_{0}=\right.$ $\left.\phi_{1}=0\right\}$, and let $\phi: \mathbb{P} \backslash L \rightarrow \mathbb{P}^{1}$ the projection with centre $L$.

Then we have a morphism $\psi: \Gamma_{2}^{\prime} \rightarrow\left(\mathbb{P}^{1} \times \mathbb{P}^{1}\right)$, the composition of the projection into $X^{\prime} \times X^{\prime}$ with the map $\phi \times \phi$.

Step IV) The points $Q_{i}$ are the fixed points of the involution $\tau$, hence if $\Delta$ is the diagonal in $\mathbb{P}^{1} \times \mathbb{P}^{1}$, then

$$
\psi^{-1}(\Delta)=\left\{\left(R_{i}, \tau R_{i}\right),\left(P_{j}^{\prime}, P_{j}^{\prime \prime}\right),\left(P_{j}^{\prime \prime}, P_{j}^{\prime}\right),\left(Q_{h}, Q_{h}\right)\right\} .
$$

As proven on page 772 of [Ca79] the points $\psi\left(Q_{h}, Q_{h}\right)$ are distinct, equivalently, the points $\phi\left(Q_{h}\right)$ are distinct, in particular $\psi$ yields a birational map from $\Gamma_{2}^{\prime}$ to its image $\Gamma^{0}$. The symmetry $\tau$ on $\Gamma_{2}^{\prime}$ induces the coordinate exchange on $\Gamma^{0}$, so that $\Gamma^{0}$ is symmetric, and of bidegree $(2 \hat{\delta}, 2 \hat{\delta})$, since $\Gamma \cap H$ consists of $2 \hat{\delta}$ ) smooth points.

Hence the intersection number of $\Gamma^{0}$ with $\Delta$ equals $4 \hat{\delta}$; but the previous calulation of the inverse image of the diagonal on the smooth curve $\Gamma_{2}^{\prime}$ shows

$$
4 \hat{\delta}=2 \hat{\delta}+2 \delta+\omega_{n}(X) \Leftrightarrow 2 \hat{\delta}=2 \delta+\omega_{n}(X) .
$$

Remark 3.4. The above proof works for any algebraically closed field of characteristic $\neq 2$.

Remark 3.2. Assume that $X \subset \mathbb{P}:=\mathbb{P}^{2 n}$ is an irreducible nondegenerate subvariety of dimension $n$ having isolated singularities $x_{1}, \ldots, x_{h}$.

We have used here Severi's original approach rather than subsequent developments, as done in [FOCV99] or [FleMan97, where the authors used the Stückrad-Vogel cycle.

It seems to us that from these works follows: one can determine, for each singular point $P=x_{i}$, a 'number of double points' $\delta_{P}$ (clearly such that $\delta_{P}=0$ if $\operatorname{Star}_{P}(X)$ has dimension $\leq 2 n-1$ ), with the property that, defining

$$
\delta=\sum_{P \in X} \delta_{P}
$$

\footnotetext{
${ }^{1} \mathrm{O}$ is chosen such that $C_{O}$ intersects $\overline{\gamma\left(X^{\prime}\right)}$ transversally at the points $\gamma\left(Q_{i}\right)$
} 
we have, in the notation of Theorem 2.1.

$$
2 \hat{\delta}=2 \delta+\omega_{n}(X)
$$

The main question is how to determine these numbers explicitly.

Example 3.3. Let $X \subset \mathbb{P}^{4}$ be the projective cone with vertex $x_{0}$ over the twisted cubic curve $D \subset \mathbb{P}^{3}$. Then $x_{0}$ is the only singular point of $X$, and clearly its tangent star is $\mathbb{P}^{4}$.

Here $D$ projects to a nodal plane cubic, hence $\hat{\delta}=1$.

Here $\omega_{2}(X)=0$, since the union of the tangent planes to $X$ has dimension 3. Hence $\delta=1$ and the normal point $x_{0}$ contributes 1 to the number of improper double points.

Replacing $D$ by a non degenerate smooth curve of genus $g$ and degree $d$ in $\mathbb{P}^{3}$, we see that again $\omega_{2}(X)=0$, hence $2 \delta_{x_{0}}=(d-1)(d-2)-2 g$.

\section{Comparing normal Sheaf And QUotient Bundle}

As in the preceding section (where we have computed the number $\delta$ of IDP' s ) we consider irreducible subvarieties $X \subset \mathbb{P}:=\mathbb{P}^{2 n}$ of dimension $n$ and with isolated singularities, which are either IDP 's or have degenerate tangent star (i.e., of dimension $\leq 2 n-1$ ).

In this section we slightly change our notation and denote the normalization of $X$ by $X^{\prime}$.

In the case where $X$ is a surface, we shall take $\tilde{X}=S$ to be a minimal resolution of singularities of $X^{\prime}$, but in general $\tilde{X}$ shall be a resolution of singularities of $X^{\prime}$, and $Y \rightarrow \tilde{X}$ a further resolution such that the Gauss map becomes a morphism

$$
\gamma: Y \rightarrow G=G r(n, 2 n) .
$$

We let $f: Y \rightarrow \mathbb{P}$ be the morphism composition of the two maps $Y \rightarrow$ $X^{\prime} \rightarrow X \subset \mathbb{P}$, and we shall use the following selfexplanatory notation: for instance $Q_{Y}=\gamma^{*} Q$.

We set $\mathcal{O}_{Y}(H):=f^{*} \mathcal{O}_{\mathbb{P}}(1)$. Then, as in Section 2,

$$
(*) 0 \rightarrow T_{Y}(-H) \rightarrow f^{*}\left(T_{\mathbb{P}}(-1)\right) \rightarrow N_{f}(-H) \rightarrow 0,
$$

and

$$
(* *) 0 \rightarrow U_{Y} / \mathcal{U}_{Y} \rightarrow f^{*}\left(T_{\mathbb{P}}(-1)\right) \rightarrow Q_{Y} \rightarrow 0 .
$$

Since $f$ is generically an immersion, $D f$ yields a sheaf inclusion $T_{Y}(-H) \subset$ $U_{Y} / \mathcal{U}_{Y}$ and associated exact sequences

$$
\begin{gathered}
0 \rightarrow T_{Y}(-H) \rightarrow U_{Y} / \mathcal{U}_{Y} \rightarrow \mathcal{F} \rightarrow 0, \\
(* * *) 0 \rightarrow \mathcal{F} \rightarrow N_{f}(-H) \rightarrow Q_{Y} \rightarrow 0,
\end{gathered}
$$

where the sheaf $\mathcal{F}$ is supported at the inverse image of the (finitely many) singular points of $X$ which are different from the IDP' s.

Definition 4.1. We define the sheaf $\mathcal{F}$ on $Y$ to be the IDP discrepancy sheaf on $Y$.

Note that $Y$ is not unique, since not always there is a minimal resolution of the closure of the Gauss map. 
Theorem 4.1. Let $X \subset \mathbb{P}^{2 n}$ be a subvariety of dimension $n$ and degree $d$ having only isolated singularities, of which $\delta$ are IDP' s, and the rest are singular points with degenerate tangent star and whose associated discrepancy sheaf $\mathcal{F}$ on $Y$ is equal to zero $\mathcal{F}=0$.

Then we have

$$
d^{2}=2 \delta+c_{n}\left(N_{f}\right)=2 \delta+c_{n}\left(f^{*}\left(T_{\mathbb{P}^{2 n}}\right)-T_{Y}\right) .
$$

If instead $\mathcal{F} \neq 0$ on $Y$, we have

$$
d^{2}=2 \delta+c_{n}\left(N_{f}\right)+c_{n}\left(N_{f}(-H)-\mathcal{F}\right)-c_{n}\left(N_{f}(-H)\right)
$$

or, equivalently,

$$
d^{2}=2 \delta+c_{n}\left(N_{f}\right)+\left[c\left(N_{f}(-H)\right) \cdot c(\mathcal{F})^{-1}\right]_{n}-c_{n}\left(N_{f}(-H)\right) .
$$

Proof. The proof of the first assertion follows from Theorem 3.3. and the argument given in Section 2 to show how to relate $c_{n}\left(Q_{Y}\right)$ with $c_{n}\left(N_{f}\right)$.

The proof of the second assertion follows with the same derivation as above plugging in the relation $c\left(Q_{Y}\right)=c\left(N_{f}(-H)\right) c(\mathcal{F})^{-1}$.

Of course, for most applications, one has to determine the sheaf $\mathcal{F}$; this can be done by explicit but sometimes lengthy calculations.

For instance, in the case of isolated hypersurface singularities

$$
X=\{x \mid F(x)=0\} \subset W:=\mathbb{C}^{n+1},
$$

the tangent plane at the smooth points $x \in X^{*}:=X \backslash\{0\}$ is the dual (annulator) subspace to the gradient at $x$. Composing the gradient map with the projection onto $\mathbb{P}\left(W^{\vee}\right)$ (the projective space of lines in the vector space $W^{\vee}$ ),

$$
\nabla F: X^{*} \rightarrow W^{\vee} \backslash\{0\}, p: W^{\vee} \backslash\{0\} \rightarrow \mathbb{P}\left(W^{\vee}\right)=: \mathbb{P}, f:=p \circ \nabla F
$$

and letting $Z$ be the closure of the graph of $f$, we see that the pull back $Q_{Z}$ of the quotient bundle is locally the direct sum of a trivial summand with $f^{*}\left(\mathcal{O}_{\mathbb{P}}(1)\right)$.

Indeed, if $L \cong \mathcal{O}_{\mathbb{P}}(-1)$ is the universal subbundle on $\mathbb{P}$, the exact sequence

$$
0 \rightarrow L \rightarrow W^{\vee} \otimes \mathcal{O}_{\mathbb{P}} \rightarrow T_{\mathbb{P}}(-1) \rightarrow 0
$$

dualizes to

$$
0 \rightarrow L^{a n n}=\Omega_{\mathbb{P}}^{1}(1) \rightarrow W \otimes \mathcal{O}_{\mathbb{P}} \rightarrow L^{\vee}=\mathcal{O}_{\mathbb{P}}(1) \rightarrow 0 .
$$

For simplicity of notation we continue to denote by $f$ the map of $Y$ to $\mathbb{P}\left(W^{\vee}\right)$ obtained from the resolution $Y \rightarrow Z$.

Since $N_{f}$ is the cokernel of $T_{Y} \rightarrow f^{*}\left(W \otimes \mathcal{O}_{\mathbb{P}}\right)$, we have exact sequences:

$$
\begin{gathered}
0 \rightarrow \mathcal{F} \rightarrow N_{f} \rightarrow f^{*}\left(\mathcal{O}_{\mathbb{P}}(1)\right) \rightarrow 0, \\
0 \rightarrow T_{Y} \rightarrow f^{*}\left(\Omega_{\mathbb{P}}^{1}(1)\right) \rightarrow \mathcal{F} \rightarrow 0 .
\end{gathered}
$$

The second sequence shows that the discrepancy sheaf $\mathcal{F}$ is a CohenMacaulay sheaf of codimension 1 on $Y$, supported on an exceptional divisor.

The first sequence instead identifies, in a neighbourhood of this divisor, the virtual sheaf $N_{f}(-H)-\mathcal{F}$ as a line bundle: $f^{*}\left(\mathcal{O}_{\mathbb{P}}(1)\right)$. 
Example 4.2. Consider a hypersurface singularity in $\mathbb{C}^{3}$, of multiplicity equal to $d$, and with tangent cone equal to the cone over a smooth plane curve $C$.

Let the local equation be

$$
X:=\left\{x=\left(x_{1}, x_{2}, x_{3}\right) \mid F(x)=0, F(x)=P_{d}(x)+\ldots\right\},
$$

so that the blow-up $Y$ of $X$ at the origin is smooth,

$$
\left.Y \subset \mathbb{C}^{3} \times \mathbb{P}^{2}, Y=\{(x, u)) \mid x=\lambda u, \lambda^{-d} F(\lambda x)=0\right\},
$$

and the Gauss map is a morphism on $Y$, which we view as a hypersurface in the universal subbundle over $\mathbb{P}^{2}$. Here $C=\{u \mid P(u)=0\}$ is identified to the execptional curve of the blow-up, and on $Y$ we have $C^{2}=-d$.

Here $U / \mathcal{U}$, at the points of $C$, equals the cone $U^{\prime}$ over the tangent line $T_{u} C$. If $\phi(t)$ is a local parametrization of $C,(t, \lambda) \mapsto \lambda \phi(t)$, hence the image of the tangent space to $Y$ is the subspace generated by $\lambda \phi^{\prime}(t)$ and $\phi(t)$, while $U^{\prime}$ is generated by $\phi^{\prime}(t)$ and $\phi(t)$. Hence the cokernel $\mathcal{F}$ is a line bundle on $C$, equal to $T C(C)=\mathcal{O}_{C}((d-2) C)$.

Therefore $c(\mathcal{F})^{-1}=1-C+C(d-2) C$, and the contribution to $2 \delta$ equals $-c_{1}\left(N_{f}\right)(-C)-(d-2) C^{2}=K_{Y} \cdot C+d(d-2)=-(d-2) C^{2}+d(d-2)=2 d(d-2)$.

The previous calculation shows that we get a positive contribution to $\delta$, equal to $d(d-2)$ : this contribution is equal to 0 if and only if we have a double point.

Remark 4.3. (a) Similar calculations could be done in order to show that the contribution we obtain equals 0 for all rational double points, which are hypersurface singularities with equation $z^{2}=f(x, y)$, where $f$ has multiplicity at most 3 , and no infinitely near triple point.

It is to observe that $Y$ is not here a minimal resolution of the singularity.

We do not pursue this calculation here, since we shall give an elementary proof in a later section that rational double points do not contribute to $\delta$.

(b) In the previous example, the Milnor number of the singularity equals $(d-1)^{3}$, while the topological Euler characteristic of the exceptional divisor $C$ is $e(C)=-d(d-3)$, hence $e(Y)-e(X)=-[d(d-3)+1]$.

Indeed, in this case the contribution equals $2\left[(d-1)^{2}-1\right]$ which is twice the Milnor number $\mu_{1}$ of a general hyperplane section through the singular point, diminished by 1 . This reminds of a formula by Piene in Pie88, end of page 25 ; however the number $1-\mu_{1}$ is not zero for rational double points $z^{2}-x^{3}-y^{3}=0$, or $z^{2}-x^{3}-y^{5}=0$ (it is -1 ). This shows that our contribution is not the local Euler obstruction.

\section{Surfaces With Rational double points}

In this section we work over any algebraically closed field $k$ of characteristic $p \geq 0$ and we show the following theorem for surfaces:

Theorem 5.1. Let $S$ be a smooth projective surface and let

$$
\mu: S \rightarrow X \subset \mathbb{P}^{4}
$$


be the minimal resolution of a surface $X$ whose singularities are only $\delta:=$ $\delta(X) I D P$ 's, and some rational double points. Then:

$$
2 \delta=d^{2}-10 d+12 \chi\left(\mathcal{O}_{S}\right)-5\left(H_{S} \cdot K_{S}\right)_{S}-2\left(K_{S}^{2}\right)_{S} .
$$

Here $H_{S}=\mu^{*} H$ for the hyperplane class $H$ of $\mathbb{P}^{4}$ and $d=\left(H_{S}^{2}\right)_{S}=\operatorname{deg} X$.

Theorem 5.1 will be applied in the following natural situation.

Let $S$ be a smooth minimal surface and let $f: S \rightarrow S^{\prime}$ be either the morphism to the canonical model for $S$ of general type, or a birational morphism onto a normal surface $S^{\prime}$ for $S$ with numerically trivial canonical class. Then $f$ is crepant and $S^{\prime}$ has only rational double points as its singularties. As $S^{\prime}$ is always embedded into some projective space $\mathbb{P}^{N}(N \geq 5)$, we may assume that $S^{\prime} \subset \mathbb{P}^{N}$. Then, as the embedded dimension of any rational double point is 3 , it follows that any general linear projection $\pi: \mathbb{P}^{N} \rightarrow \mathbb{P}^{4}$, as explained above, induces a morphism

$$
\left.\pi\right|_{S^{\prime}}: S^{\prime} \rightarrow X:=\pi\left(S^{\prime}\right) \subset \mathbb{P}^{4}
$$

such that $\left.\pi\right|_{S^{\prime}}$ is isomorphic at all rational double points of $S^{\prime}$ and $X$ has only these rational double points and finitely many improper double points. So, the morphism

$$
\left.\pi\right|_{S^{\prime}} \circ f: S \rightarrow X \subset \mathbb{P}^{4}
$$

satisfies the assumption made in Theorem 5.1. In fact, this gives, among other things, a generalization of a result of the first author Ca97, Prop. 6.2, Cor. 6.3] see also [Ca16, Theorem 0.2], and an affirmative answer to the conjecture made there.

Corollary 5.2. Let $S$ be a minimal smooth surface of general type with $p_{g}(S)=5$ such that the canonical map

$$
\Phi_{\left|K_{S}\right|}: S \rightarrow S^{\prime} \subset \mathbb{P}^{4}
$$

is a morphism with image isomorphic to the canonical model $S^{\prime}$ of $S$. Then, setting $d:=\left(K_{S}^{2}\right)_{S}$,

$$
12 \chi\left(\mathcal{O}_{S}\right)=(17-d) d .
$$

In particular, $S^{\prime}$ is then a complete interesection of type $(2,4)$ or $(3,3)$, if either the base field is of characteristic 0 or the characteristic of the field $k$ is odd and $h^{1}\left(\mathcal{O}_{S}\right)=0$.

Proof. We have $\delta=0,\left(H_{S}^{2}\right)_{S}=\left(K_{S}^{2}\right)_{S}=d$ by our assumption. So, the first equality follows from Theorem 5.1. The last statement then follows from the proof of Ca16, Theorem 0.2]. Note that, in positive odd characteristic case, we have $h^{1}\left(m K_{S}\right)=0$ for all $m \geq 1$ by our assumption (for $m=1$ ) and [Ek88, Theorem 1.7] (for $m \geq 2$ ); hence $\chi\left(\mathcal{O}_{S}\right)=6$ by our assumption, and the rest of the proof follows as in Ca97, page 41.

Problem 5.1. Are there exceptions to the above statement that $S^{\prime}$ is a complete intersection in odd positive characteristic for $H^{1}\left(\mathcal{O}_{S}\right) \neq 0$, or in characteristic $p=2$ ?

The above corollary was actually an initial motivation for our study.

The following fact will be used frequently in the proof of Theorem 5.1 
Lemma 5.3. Let $\pi: V \rightarrow W$ be the blow up at $Q \in W$ of a smooth projective variety $W$ of dimension 4 , let $E=\pi^{-1}(Q) \simeq \mathbb{P}^{3}$ be the exceptional divisor and let $P \simeq \mathbb{P}^{2}$ be a plane in $E \simeq \mathbb{P}^{3}$. Then, in the Chow ring of $V$, we have:

$$
c_{1}(V)=\pi^{*} c_{1}(W)-3 E, c_{2}(V)=\pi^{*} c_{2}(W)-2 P,(P . P)_{V}=-1 .
$$

Proof. As $\pi$ is the blow up at a smooth point of a 4 -fold, we have that the Chow ring $A(V)$ of $V$ is generated by the pull back of the Chow ring of $W$ and by the exceptional divisor.

We have moreover $E^{2}=\left.E\right|_{E}=-P$. Thus

$$
(P . P)_{V}=\left(E^{4}\right)_{V}=\left(\left(\left.E\right|_{E}\right)^{3}\right)_{E}=\left((-P)^{3}\right)_{E}=-1 .
$$

This proves the last formula. As $c_{1}(V)=-K_{V}$ and $c_{1}(W)=-K_{W}$, the first formula is nothing but the canonical bundle formula under the blow up.

Let us show the second formula. We have $A^{2}(V)=\pi^{*} A^{2}(W) \oplus \mathbb{Z}[P]$ and, evaluating on two dimensional cycles avoiding the exceptional divisor, we infer that we can write $c_{2}(V)=\pi^{*} c_{2}(W)+a P$. In order to determine $a$, consider the exact sequence

$$
\left.0 \rightarrow T_{P} \rightarrow T_{V}\right|_{P} \rightarrow N_{V / P} \rightarrow 0 \text {. }
$$

By functoriality of the Chern class, we have

$$
\text { (A) }\left(c_{2}(V) \cdot P\right)_{V}=\left(c_{1}(P) \cdot c_{1}\left(N_{V / P}\right)\right)_{P}+c_{2}(P)+c_{2}\left(N_{V / P}\right) \text {. }
$$

The left hand side is then

$$
\left(c_{2}(V) \cdot P\right)_{V}=\left(\pi^{*} c_{2}(W)+a P \cdot P\right)=a(P \cdot P)=-a .
$$

We compute the right hand side. Note that $c_{1}(P)=-K_{P}=3 l$ in the Chow ring, where $l$ is a line in $P \simeq \mathbb{P}^{2}$. We have

$$
\text { (B) }\left.c_{1}(V)\right|_{P}=c_{1}(P)+c_{1}\left(N_{V / P}\right)=3 l+c_{1}\left(N_{V / P}\right)
$$

from the exact sequence above. Note that, as $l, P \subset E$, we have

$$
\left(\left.E\right|_{P} . l\right)_{P}=\left(\left.E\right|_{E} \cdot l\right)_{E}=(-P . l)_{E}=-1 .
$$

In particular, $\left.E\right|_{P}=-l$ in the Chow ring of $P$. Combining this with the first equality, we have

$$
\left.c_{1}(V)\right|_{P}=\left.\left(\pi^{*} c_{1}(W)-3 E\right)\right|_{P}=-\left.3 E\right|_{P}=3 l .
$$

Hence $c_{1}\left(N_{V / P}\right)=0$ by (B). Since $P \subset V$ is a smooth subvariety of codimension $2=4 / 2$, by the selfintersection formula and the last formula $\left(P^{2}\right)_{V}=$ -1 , we have $c_{2}\left(N_{V / P}\right)=\left(P^{2}\right)_{V}=-1$. As $P \simeq \mathbb{P}^{2}$, we have $c_{2}(P)=3$. Substituting everything into formula (A), we obtain $-a=0+3-1$, hence $a=-2$ as claimed.

The rest of this section is devoted to prove Theorem 5.1,

Proof. Let $x_{j}$, for $1 \leq j \leq \delta$, be the IDP's of $X$. Consider the blow up

$$
f: V_{1} \rightarrow \mathbb{P}^{4}
$$

of $\mathbb{P}^{4}$ at the points $x_{j}, 1 \leq j \leq \delta$.

Let $S_{0}$ be the proper transform of $X$ and $\left.f\right|_{S_{0}}: S_{0} \rightarrow X$ be the induced morphism. Then $\left.f\right|_{S_{0}}$ is an isomorphism except over the points $x_{j}, S_{0}$ is smooth over the points $x_{j}$ and $\left.f\right|_{S_{0}} ^{-1}\left(x_{j}\right)$ consists of two $(-1)$-curves, i.e., 
two smooth rational curves of self-intersection number $(-1)$. We denote these two curves by $l_{j 1}$ and $l_{j 2}$.

Next, we are going to take the minimal embedded resolution of $S_{0} \subset V_{0}$ via a sequence of point blow-ups. Let

$$
g_{1}: V_{1} \rightarrow V_{0}
$$

be the blow up of $V_{0}$ at a rational double point, say $P$, of $S_{0}$ and define $S_{1}$ to be the proper transform of $S_{0}$ under $g_{1}$. Then we have an induced morphism $\left.g_{1}\right|_{S_{1}}: S_{1} \rightarrow S_{0}$ which is the same as the blow up of $S_{0}$ at the maximal ideal of $P$. The special properties of rational double points that we need is that the canonical divisor $K_{S_{0}}$ is Cartier, $S_{1}$ continues to have only rational double points as singularities, and the morphism $\left.g_{1}\right|_{S_{1}}$ is crepant, i.e., $\left(\left.g_{1}\right|_{S_{1}}\right)^{*} K_{S_{0}}=K_{S_{1}}$ (see Ar66]). Now we choose an embedded resolution of $S_{0} \subset V_{0}$ inductively by

$$
g_{i}: V_{i} \rightarrow V_{i-1},\left.g_{i}\right|_{S_{i}}: S_{i} \rightarrow S_{i-1}
$$

$(i=1,2, \ldots, n)$, where $g_{i}$ is the blow up of $S_{i-1}$ at some (singular) rational double point, $S_{i}$ is the proper transform of $S_{i-1}$, and $S_{n}$ is smooth.

We set

$$
\tilde{S}:=S_{n},\left.g\right|_{\tilde{S}}: \tilde{S} \rightarrow S_{0},
$$

where $\left.g\right|_{\tilde{S}}$ is the morphism induced by $g: V_{n} \rightarrow V_{0}$, the composition of the $g_{i}{ }^{\prime}$ s. We also denote respective proper transforms of $l_{j 1}, l_{j 2}$ on $\tilde{S}$ by the same letter. This is harmless for us, as they are disjoint from the exceptional divisors of $g$.

Clearly $\tilde{S}$ is birational to the original $S$ in our Theorem 5.1. Recall that we started from the birational morphism $\mu: S \rightarrow X \subset \mathbb{P}^{4}$. Indeed, by our assumption, the birational map $\nu:=\left.\left.\mu \circ f\right|_{S_{0}} \circ g\right|_{\tilde{S}}: \tilde{S} \rightarrow S$ is the contraction morphism of the $2 \delta(-1)$-curves $l_{j 1}, l_{j 2}$. We denote the exceptional divisor of $g_{i}: V_{i} \rightarrow V_{i-1}$ by $E_{i}$ and let $P_{i} \simeq \mathbb{P}^{2}$ be a plane of $E_{i} \simeq \mathbb{P}^{3}$.

Lemma 5.4. Under the above setting,

$$
S_{i}=g_{i}^{*} S_{i-1}-2 P_{i},\left(S_{i} \cdot P_{i}\right)_{V_{i}}=2
$$

in the Chow ring of $V_{i}$.

Proof. Since $A^{2}\left(V_{i}\right)=g_{i}^{*} A^{2}\left(V_{i-1}\right) \oplus \mathbb{Z} P_{i}$, one can write $S_{i}=g_{i}^{*} S_{i-1}+a P_{i}$ for some integer $a$. Then $\left(S_{i} \cdot P_{i}\right)_{V_{i}}=a\left(P_{i} \cdot P_{i}\right)_{V_{i}}=-a$ by the formula above and Lemma 5.3. On the other hand, as $g_{i}$ is the blow up at a rational double point of $S_{i}$, it follows that $\left.S_{i}\right|_{E_{i}}$ is a plane conic curve in $E_{i} \simeq \mathbb{P}^{3}$, while $P_{i}$ is a plane in $E_{i} \simeq \mathbb{P}^{3}$. Thus

$$
\left(S_{i} \cdot P_{i}\right)_{V_{i}}=\left(\left.S_{i}\right|_{E_{i}} \cdot P_{i}\right)_{E_{i}}=2 .
$$

Therefore $a=-2$ as claimed.

We set

$$
\chi_{i}:=\left(c_{2}\left(V_{i}\right) \cdot S_{i}\right)_{V_{i}}+\left(\left.c_{1}\left(V_{i}\right)\right|_{S_{i}} \cdot K_{S_{i}}\right)_{S_{i}}+2\left(K_{S_{i}}^{2}\right)_{S_{i}}-\left(S_{i} \cdot S_{i}\right)_{V_{i}}
$$

for all integers $i$ with $0 \leq i \leq n-1$.

Lemma 5.5. In the above setting, $12 \chi\left(\mathcal{O}_{S}\right)=\chi_{n-1}$. 
Proof. Since $S_{n} \subset V_{n}$ are both smooth, we have the exact sequence

$$
\left.0 \rightarrow T_{S_{n}} \rightarrow T_{V_{n}}\right|_{S_{n}} \rightarrow N_{V_{n} / S_{n}} \rightarrow 0
$$

and we obtain

$$
\text { (A) : }\left.c_{1}\left(V_{n}\right)\right|_{S_{n}}=c_{1}\left(S_{n}\right)+c_{1}\left(N_{V_{n} / S_{n}}\right)=-K_{S_{n}}+c_{1}\left(N_{V_{n} / S_{n}}\right) .
$$

By Lemma 5.3, we compute the left hand side of (A) as

$$
\left.c_{1}\left(V_{n}\right)\right|_{S_{n}}=\left(\left.g_{n}\right|_{S_{n}}\right)^{*}\left(\left.c_{1}\left(V_{n-1}\right)\right|_{S_{n-1}}\right)-3 R_{n},
$$

where $R_{n}:=\left.E_{n}\right|_{S_{n}}$. Substituting this into (A), we obtain

$$
\text { (B) : } c_{1}\left(N_{V_{n} / S_{n}}\right)=\left(\left.g_{n}\right|_{S_{n}}\right)^{*}\left(\left.c_{1}\left(V_{n-1}\right)\right|_{S_{n-1}}\right)-3 R_{n}+K_{S_{n}} .
$$

Again from the normal bundle sequence, we obtain

$$
\text { (C) : }\left.c_{2}\left(V_{n}\right)\right|_{S_{n}}=c_{1}\left(N_{V_{n} / S_{n}}\right) c_{1}\left(S_{n}\right)+c_{2}\left(S_{n}\right)+c_{2}\left(N_{V_{n} / S_{n}}\right) .
$$

Using Lemma 5.3, we compute the left hand side of (C) as

$$
\left.c_{2}\left(V_{n}\right)\right|_{S_{n}}=\left(g_{n}^{*} c_{2}\left(V_{n-1}\right) \cdot S_{n}\right)_{V_{n}}-2\left(P_{n} \cdot S_{n}\right)_{V_{n}}=\left(c_{2}\left(V_{n-1}\right) \cdot S_{n-1}\right)_{V_{n-1}}-4 \text {. }
$$

We compute now each term of the right hand side of (C). Since $\left.g_{n}\right|_{S_{n}}$ is crepant, we have $\left(R_{n} \cdot K_{S_{n}}\right)_{S_{n}}=0$. Thus, by using (B), we compute

$$
\begin{gathered}
\left(c_{1}\left(N_{V_{n} / S_{n}}\right) \cdot c_{1}\left(S_{n}\right)\right)_{S_{n}}=\left(\left(\left.g_{n}\right|_{S_{n}}\right)^{*}\left(\left.c_{1}\left(V_{n-1}\right)\right|_{S_{n-1}}\right) \cdot\left(-K_{S_{n}}\right)\right)_{S_{n}}-\left(K_{S_{n}}^{2}\right)_{S_{n}}= \\
=\left(\left.c_{1}\left(V_{n-1}\right)\right|_{S_{n-1}} \cdot-K_{S_{n-1}}\right)_{S_{n-1}}-\left(K_{S_{n-1}}^{2}\right)_{S_{n-1}} .
\end{gathered}
$$

Using Noether's formula for the smooth projective surface $S_{n}$, the fact that $\chi\left(\mathcal{O}_{S}\right)$ is invariant under birational smooth modification, and the fact that $\left.g_{n}\right|_{S_{n}}$ is crepant, we compute

$$
c_{2}\left(S_{n}\right)=12 \chi\left(\mathcal{O}_{S_{n}}\right)-\left(K_{S_{n}}^{2}\right)_{S_{n}}=12 \chi\left(\mathcal{O}_{S}\right)-\left(K_{S_{n-1}}^{2}\right)_{S_{n-1}} .
$$

By using again the selfintersection formula $c_{2}\left(N_{V_{n} / S_{n}}\right)=\left(S_{n}^{2}\right)_{V_{n}}$ and the formula $S_{n}=g_{n}^{*} S_{n-1}-2 P_{n}$ (Lemma 5.4), we have

$$
c_{2}\left(N_{V_{n} / S_{n}}\right)=\left(S_{n} \cdot S_{n}\right)_{V_{n}}=\left(S_{n-1} \cdot S_{n-1}\right)_{V_{n-1}}-4 .
$$

Substituting all into equation $(\mathrm{C})$, we obtain that

$$
\begin{gathered}
\left(c_{2}\left(V_{n-1}\right) \cdot S_{n-1}\right)_{V_{n-1}}-4=\left(\left.c_{1}\left(V_{n-1}\right)\right|_{S_{n-1}} \cdot-K_{S_{n-1}}\right)_{S_{n-1}}-2\left(K_{S_{n-1}}^{2}\right)_{S_{n-1}} \\
+\left(S_{n-1} \cdot S_{n-1}\right)_{V_{n-1}}-4+12 \chi\left(\mathcal{O}_{S}\right) .
\end{gathered}
$$

By the definition of $\chi_{i}$, this equality is equivalent to $12 \chi\left(\mathcal{O}_{S}\right)=\chi_{n-1}$. This completes the proof of Lemma [5.5.

Lemma 5.6. The number $\chi_{i}$ is independent of $i$, for all $0 \leq i \leq n-1$.

Proof. Let $i \geq 1$. Then by Lemma 5.3 and Lemma 5.4, we have

$$
\begin{aligned}
\left(c_{2}\left(V_{i}\right) \cdot S_{i}\right)_{V_{i}} & =\left(\left(g_{i}^{*} c_{2}\left(V_{i-1}\right)-2 P_{i}\right) \cdot\left(g_{i}^{*} S_{i-1}-2 P_{i}\right)\right)_{V_{i}}=\left(c_{2}\left(V_{i-1}\right) \cdot S_{i-1}\right)_{V_{i-1}}-4, \\
\left(S_{i} \cdot S_{i}\right)_{V_{i}} & =\left(\left(g_{i}^{*} S_{i-1}-2 P_{i}\right) \cdot\left(g_{i}^{*} S_{i-1}-2 P_{i}\right)\right)_{S_{i}}=\left(S_{i-1} \cdot S_{i-1}\right)_{V_{i-1}}-4 .
\end{aligned}
$$

Using Lemma 5.3 and observing that $K_{S_{i}}=\left(\left.g_{i}\right|_{S_{i}}\right)^{*} K_{S_{i-1}}$, we have $\left(\left.c_{1}\left(V_{i}\right)\right|_{S_{i}} \cdot K_{S_{i}}\right)_{S_{i}}=\left(\left.\left(g_{i}^{*} c_{1}\left(V_{i-1}\right)-2 P_{i}\right)\right|_{S_{i}} \cdot\left(\left.g_{i}\right|_{S_{i}}\right)^{*} K_{S_{i-1}}\right)=\left(\left.c_{1}\left(V_{i-1}\right)\right|_{S_{i-1}} \cdot K_{S_{i-1}}\right)_{S_{i-1}}$, and $\left(K_{S_{i}}^{2}\right)_{S_{i}}=\left(K_{S_{i-1}}^{2}\right)_{S_{i-1}}$. Substituting all these equalities into the formula in Lemma 5.6, we obtain that $\chi_{i}=\chi_{i-1}$. This implies the result. 
Lemma 5.7. In the above setting,

$$
12 \chi\left(\mathcal{O}_{S}\right)=\left(c_{2}\left(V_{0}\right) \cdot S_{0}\right)_{V_{0}}+\left(\left.c_{1}\left(V_{0}\right)\right|_{S_{0}} \cdot K_{S_{0}}\right)_{S_{0}}+2\left(K_{S_{0}}^{2}\right)_{S_{0}}-\left(S_{0} \cdot S_{0}\right)_{V_{0}} .
$$

Proof. By Lemma 5.5 and Lemma 5.4, we have $12 \chi\left(\mathcal{O}_{S}\right)=\chi_{n-1}=\chi_{0}$. The right hand side of the formula in Lemma 5.7 is by definition equal to $\chi_{0}$. This completes the proof.

Lemma 5.8. In the above situation, and with the usual notation where $H_{S}$ is the pullback of the hyperplane class $H$ of $\mathbb{P}^{4}$ under $\mu: S \rightarrow X \subset \mathbb{P}^{4}$ and $d=\left(H_{S}^{2}\right)_{S}=\operatorname{deg} X$, we have:

$$
12 \chi\left(\mathcal{O}_{S}\right)=10 d-d^{2}+2\left(K_{S}^{2}\right)_{S}+5\left(H_{S} \cdot K_{S}\right)+2 \delta .
$$

Proof. We shall prove the formula by computing each term of the right hand side of the equation in Lemma 5.7

Let $F_{j} \simeq \mathbb{P}^{3}$ be the exceptional divisor of $f: V_{0} \rightarrow \mathbb{P}^{4}$ over $x_{j}$ and let $R_{j} \simeq \mathbb{P}^{2}$ be a plane in $F_{j}$.

Then $c_{*}\left(\mathbb{P}^{4}\right)=(1+H)^{5}$ in the Chow ring of $\mathbb{P}^{4}$, and

$$
\begin{gathered}
c_{1}\left(V_{0}\right)=f^{*} c_{1}\left(\mathbb{P}^{4}\right)-3 \sum_{j=1}^{\delta} F_{j}, \\
c_{2}\left(V_{0}\right)=f^{*} c_{2}\left(\mathbb{P}^{4}\right)-2 \sum_{j=1}^{\delta} R_{j},\left(R_{j} \cdot R_{j}\right)_{V_{0}}=-1
\end{gathered}
$$

by Lemma 5.3 , and

$$
S_{0}=f^{*} X-2 \sum_{j=1}^{\delta} R_{j}=f^{*}\left(d H^{2}\right)-2 \sum_{j=1}^{\delta} R_{j}
$$

in the Chow ring of $V_{0}$. Hence

$$
\begin{gathered}
\left(c_{2}\left(V_{0}\right) \cdot S_{0}\right)_{V_{0}}=\left(\left(10 f^{*} H^{2}-2 \sum_{j=1}^{\delta} R_{j}\right) \cdot\left(d f^{*} H^{2}-2 \sum_{j=1}^{\delta} R_{j}\right)_{V_{0}}=10 d-4 \delta,\right. \\
\left(S_{0} \cdot S_{0}\right)=\left(\left(d f^{*}\left(H^{2}\right)-2 \sum_{j=1}^{\delta} R_{j}\right)^{2}\right)=d^{2}-4 \delta .
\end{gathered}
$$

As $g: \tilde{S} \rightarrow S_{0}$ is crepant and $\tilde{S} \rightarrow S$ contracts exactly $2 \delta$ disjoint (-1)curves, we obtain

$$
\left(K_{S_{0}}^{2}\right)_{S_{0}}=\left(K_{\tilde{S}}^{2}\right)_{\tilde{S}}=\left(K_{S}^{2}\right)_{S}-2 \delta .
$$

Finally we compute $\left(\left.c_{1}\left(V_{0}\right)\right|_{S_{0}} \cdot K_{S_{0}}\right)_{S_{0}}$. First of all, we have

$$
\begin{gathered}
\left(\left.c_{1}\left(V_{0}\right)\right|_{S_{0}} \cdot K_{S_{0}}\right)_{S_{0}}=\left(\left.(f \circ g)^{*} c_{1}\left(\mathbb{P}^{4}\right)\right|_{\tilde{S}} \cdot K_{\tilde{S}}\right)_{\tilde{S}} \\
\left.\left.=\left(5 H_{\tilde{S}}-\left.3 \sum_{j=1}^{\delta} F_{j}\right|_{\tilde{S}}\right) \cdot K_{\tilde{S}}\right)_{\tilde{S}}=\left(5 H_{\tilde{S}} \cdot K_{\tilde{S}}\right)_{\tilde{S}}-3 \sum_{j=1}^{\delta}\left(l_{j 1}+l_{j 2}\right) \cdot K_{\tilde{S}}\right)_{\tilde{S}} \cdot
\end{gathered}
$$

Here $F_{j}$ is the proper transform of the exceptional divisor $f^{-1}\left(x_{j}\right)$ of $f$ and we used the fact that $\left.g\right|_{\tilde{S}}: \tilde{S} \rightarrow S_{0}$ is crepant for the second equality so there appears no exceptional divisor of $g_{i}$ in the formula. As $l_{j 1}$ and $l_{j 2}$ are $(-1)$-curves on a smooth surface $\tilde{S}$, we have $\left(l_{j 1} \cdot K_{\tilde{S}}\right)_{\tilde{S}}=\left(l_{j 2} \cdot K_{\tilde{S}}\right)_{\tilde{S}}=-1$. 
Moreover, since the morphism $\nu: \tilde{S} \rightarrow S$ defined above is the contraction of exactly $2 \delta(-1)$-curves $l_{j 1}$ and $l_{j 2}$, it follows that

$$
\left(H_{\tilde{S}} \cdot K_{\tilde{S}}\right)_{\tilde{S}}=\left(\nu^{*} H_{S} \cdot\left(\nu^{*} K_{S}+\sum_{j=1}^{\delta}\left(l_{j 1}+l_{j 2}\right)\right)\right)_{\tilde{S}}=\left(H_{S} \cdot K_{S}\right)_{S} \cdot
$$

Substituting these two formulae into the last formula for $\left(\left.c_{1}\left(V_{0}\right)\right|_{S_{0}} \cdot K_{S_{0}}\right)$, we obtain that $\left(\left.c_{1}\left(V_{0}\right)\right|_{S_{0}} \cdot K_{S_{0}}\right)=5\left(H_{S} \cdot K_{S}\right)$. Substituting the four formulae that we obtained for $\left(c_{2}\left(V_{0}\right) \cdot S_{0}\right)_{V_{0}},\left(\left.c_{1}\left(V_{0}\right)\right|_{S_{0}} \cdot K_{S_{0}}\right)_{S_{0}},\left(K_{S_{0}}^{2}\right)_{S_{0}}$ and $\left(S_{0} \cdot S_{0}\right)_{V_{0}}$ inside the formula in Lemma 5.7, we obtain

$$
12 \chi\left(\mathcal{O}_{S}\right)=10 d-4 \delta+5\left(H_{S} \cdot K_{S}\right)+6 \delta+2\left(K_{S}^{2}\right)-4 \delta-d^{2}+4 \delta .
$$

Simplifying the right hand side, we obtain

$$
12 \chi\left(\mathcal{O}_{S}\right)=10 d-d^{2}+2\left(K_{S}^{2}\right)_{S}+5\left(H_{S} \cdot K_{S}\right)_{S}+2 \delta,
$$

as claimed.

This completes the proof of Theorem 5.1

\section{Double Point Formulae Via Symplectic APproximations.}

We introduce, inspired by a concept introduced by Kodaira in [Kod65, a class of isolated singularities.

Definition 6.1. An n-dimensional isolated singularity $O \in X \subset \mathbb{C}^{2 n}$ is said to be quasi-improper-multiple point $=$ QIMP, if $X$ consists of $r$ smooth branches $X_{1}, \ldots, X_{r}$ passing through the origin $O$.

To explain the notion, one can take a good projection yielding a splitting $\mathbb{C}^{2 n}=\mathbb{C}^{n} \oplus \mathbb{C}^{n}$, so that

$$
X_{i}=\left\{(x, y) \in \mathbb{C}^{n} \oplus \mathbb{C}^{n} \mid y=F_{i}(x)\right\} .
$$

Clearly the intersection points $X_{i} \cap X_{j}$ correspond to the set

$$
\Gamma_{i j}:=\left\{x \in \mathbb{C}^{n} \mid F_{i}(x)-F_{j}(x)=0\right\} .
$$

The hypothesis of isolated singularities amounts to the requirement that $0 \in \mathbb{C}^{n}$ is isolated in the locus $\Gamma_{i j}$, and, setting $\mathcal{O}:=\mathcal{O}_{\mathbb{C}^{n}, 0}$, we consider the intersection multiplicity

$$
m_{i j}:=\operatorname{dim}_{\mathbb{C}}\left(\mathcal{O} /\left(F_{i}-F_{j}\right) \mathcal{O}^{n}\right),
$$

where $F_{i}-F_{j}$ is considered as a $1 \times n$ matrix. It is clear that, for a generic perturbation of the branches $X_{i}$, the isolated singularity deforms to

$$
\delta_{O}:=\sum_{i<j} m_{i j}
$$

IDP's. This is why we shall say that $\delta_{O}$ is the local number of IDP's.

Theorem 6.1. Let $X \subset \mathbb{P}^{2 n}$ be a complex variety with isolated singularities, of which

(1) $h$ are quasi-improper-multiple points, such that the sum of the local numbers of IDP's equals $\delta$,

(2) the other singular points are normal and locally smoothable. 
Then $X$ admits a global smoothing to a symplectic immersed manifold $M \subset \mathbb{P}^{2 n}$, with exactly $\delta$ IDP's, and we have, if $f: M^{\prime} \rightarrow M$ is the immersion, that

$$
d^{2}=2 \delta+e\left(N_{f}\right)
$$

where $e$ is the Euler class of the oriented normal bundle to the map.

Proof. The result follows from Theorem 1.2 of [Ca09], and a local deformation at the non normal singularities, showing that we can deform $X$ to a symplectic immersed manifold $M \subset \mathbb{P}^{2 n}$, with exactly $\delta$ IDP's.

Letting $f: M^{\prime} \rightarrow M$ be the immersion, we calculate the self intersection $d^{2}$ of $M$ in $\mathbb{P}^{2 n}$ (in the same way as explained in the introduction) as $2 \delta$ plus the self intersection number of the zero section in the normal bundle $N_{f}$ (which maps via the exponential map onto a neighbourhood of $X$ ).

Remark 6.2. A special case is the one where the normal singularities are isolated hypersurface singularities.

The following was the original idea which led us to realize that the Severi double point formula for smooth surfaces (i.e., smooth outside of the IDP's) holds verbatim if we also allow rational double points, at least over $\mathbb{C}$.

In fact, for surface rational double points over $\mathbb{C}$, one has the fortunate coincidence that $M^{\prime}$ coincides with the minimal resolution $\pi: S \rightarrow X$, and then one has only to observe that $\pi$ and $f$ are a differentiable deformation of each other, which can be taken as the identity outside the inverse image $S_{B}$ of a sufficiently small neighbourhood $B$ of the normal singularities.

In this case, a possible way to show the equality between $e\left(N_{f}\right)$ and $c_{2}\left(N_{\pi}\right)$, following the arguments of [Ca09], could be to argue that the Euler number of $N_{f}$ is obtained by integrating the top Chern form of $N_{\pi}$ outside $S_{B}$, plus adding an integral on the Milnor fibre $M_{B}^{\prime}$, diffeomorphic to the neighbourhood $S_{B}$ of the exceptional divisor: this integral should yield the top Chern class integral on the tangent bundle of $S_{B}$, and then the desired equality would follow.

Acknowledgements: The authors would like to thank Alex Dimca for useful discussions, and Paolo Aluffi for answering the first author's email queries.

\section{REFERENCES}

[A194] P. Aluffi, MacPherson's and Fulton's Chern classes of hypersurfaces, Internat. Math. Res. Notices 11 (1994) 455-465.

[Al16] P. Aluffi, Projective duality and a Chern-Mather involution, arXiv 1601.05427.

[Al18] P. Aluffi, The Chern-Schwartz-MacPherson class of an embeddable scheme, arXiv 1805.1111.

[Ar66] M. Artin, On isolated rational singularities of surfaces, Amer. J. Math. 88 (1966) 129-136.

[BSS09] J.P. Brasselet, J. Seade, T. Suwa, Vector fields on singular varieties., Lecture Notes in Mathematics 1987. Berlin: Springer xx, 225 p. (2009).

[Ca79] F. Catanese, On Severi's proof of the double point formula, Comm. Algebra 7 (1979) 763-773.

[Ca97] F. Catanese, Homological algebra and algebraic surfaces, Algebraic geometry Santa Cruz 1995, 3-56, Proc. Sympos. Pure Math. 62 Part 1, Amer. Math. Soc., Providence, RI, 1997. 
[Ca09] F. Catanese, Canonical symplectic structures and deformations of algebraic surfaces, Comm. Contemp. Math. 11, N. 3 (2009) 481-493.

[Ca16] F. Catanese, Canonical surfaces of higher degree, Rend. Circ. Mat. Palermo 66 (2017) 43-51.

[Ca19] F. Catanese, Canonical maps of hypersurfaces in Abelian varieties, in preparation.

[deV75] A. Van de Ven, On the embedding of abelian varieties in projective spaces, Annali di Matematica Pura ed Applicata 103 (1975) 127-129.

[Ek88] T. Ekedahl, Canonical models of surfaces of general type in positive characteristic, Inst. Hautes Études Sci. Publ. Math. 67 (1988) 97-144.

[FOCV99] H. Flenner, L. O' Carroll, W. Vogel Joins and Intersections, Springer Monographs in Mathematics. Berlin: Springer. vi, 307 p. (1999).

[FleMan97] H. Flenner, M. Manaresi Intersections of Projective Varieties and Generic Projections, Manuscripta Math. 92, 273-286 (1997).

[Fu84] W. Fulton, Intersection Theory, Ergebnisse der Mathematik und ihrer Grenzgebiete, 2 Springer-Verlag, Berlin, (1984).

[FL77] W. Fulton, D. Laksov, Residual intersections and the double point formula, Real and complex singularities (Proc. Ninth Nordic Summer School/NAVF Sympos. Math., Oslo, 1976), 171-177. Sijthoff and Noordhoff, Alphen aan den Rijn, (1977).

[GH78] P. Griffiths, J. Harris, Principles of algebraic geometry, Pure and Applied Mathematics. Wiley-Interscience, New York, (1978).

[Jo78] K. Johnson, Immersion and embedding of projective varieties, Acta Math. 140 (1978) 49-74.

[Ka19] K. Kani, On the embeddability of hyperkähler fourfolds into the projective space of dimension 8, (master thesis, U. Tokyo), 2019, preprint.

[Kod65] K. Kodaira, On characteristic systems of families of surfaces with ordinary singularities in a projective space, Amer. J. Math. 87 (1965) 227-256.

[LMS75] A. T. Lascu, D. Mumford, D. B. Scott, The self-intersection formula and the "formule-clef". Math. Proc. Cambridge Philos. Soc. 78 (1975) 117-123.

[Ma74] R. D. MacPherson, Chern classes for singular algebraic varieties, Ann. of Math. 100 (1974) 423-432.

[Na95] J. F. Nash, Arc structure of singularities, Duke Math. J. 81 (1995) 31-38.

[Pie88] R. Piene, Cycles polaires et classes de Chern pour les variétés projectives singulières. [Polar cycles and Chern classes for singular projective varieties] Introduction A la thèorie des singularités, II, 7-34, Travaux en Cours, 37, Hermann, Paris, (1988).

[Se02] F. Severi, Sulle intersezioni delle varietá algebriche e sopra i loro caratteri e singolaritá proiettive, Mem. Accad. Scienze di Torino, S. II 52 (1902) 61-118; also in Memorie Scelte I, Zuffi (1950) (Bologna).

[SUV97] A. Simis, B. Ulrich, W. V. Vasconcelos, Tangent star cones, J. Reine Angew. Math. 483 (1997) 23-59.

Lehrstuhl Mathematik VIII, Mathematisches Institut der Universität Bayreuth, NW II, Universitätsstr. 30, 95447 Bayreuth, And Korea Institute for Advanced Study, Hoegiro 87, Seoul, 133-722, Korea

E-mail address: Fabrizio.Catanese@uni-bayreuth.de

Mathematical Sciences, the University of Tokyo, Meguro Komaba 3-8-1, Tokyo, Japan, and Korea Institute for Advanced Study, Hoegiro 87, Seoul, 133-722, KOREA

E-mail address: oguiso@ms.u-tokyo.ac.jp 